\title{
Analysis of Gearbox Housing Dynamic Performance under Periodic Excitation
}

\author{
Xing-Yu Wang, Ze-Fei Wang, Long Cheng \\ CRRC, Changchun Railway Vehicle Company Ltd., \\ Changchun 130062, Jilin China \\ E-mail: maklcewxy@163.com
}

\begin{abstract}
In order to analyze characteristics of gearbox housing under polygonal wheel excitation in high-speed train. The wheel circumference irregular was simulated by the method of multi-harmonic excitation at first, and the meshing force generated in the inner of gearbox has also been considered. A non-linear dynamics model of the whole vehicle was established and the dynamic performance of polygon wheel influence on housing has also been simulated. The dynamic force was loaded to the finite element model of gearbox housing as well as analyzed the dynamic response of housing. The results showed that the wheel circumference irregular will increase the gear transmission system responses and affect the life expectancy of housing. The polygonal frequency also appeared in the dynamic response of gearbox housing. Based on these research results that during the structure design of gearbox, it should make full use of interlaced frequency, so that it can reduce partial stress of housing which originated be resonance.
\end{abstract}

Keywords-polygonal wheel; gearbox; periodic excitation; high-speed train

\section{PREFACE}

Gearbox is the key component of power transmission of high-speed train, typical bogie suspension type of gearbox is installed on the axle through one end, and the opposite end is installed on the bogie frame via support arm(C-type bracket), as shown in Figure1. The gearbox housing often be seen as the key part in gearbox system, during the process of high-speed train operating, on the one hand, the housing have to bear load from wheel-rail vibration, on the other hand, due to the cyclic variation of meshing stiffness during gear pair meshing process, vibration caused by internal dynamic excitation will be transferred to housing through axle and bearing. Under the impact combined effect of external and internal incentives, this kind of impact will lead housing has to bear very complex alternating load, moreover, stress change of housing will affect the safety and life of the gear transmission system directly[1].

Polygonal wheel is a common wearing form of railway vehicle`s wheels, which often appear in the operating process, the concrete manifestation is that wheel tread will form periodic irregularity along the circumferential direction, consequently, the wheel-rail system will produce periodic excitation and transfer vibration to bogie, this kind of excitation will cause vibration increasing remarkably, more seriously, it will affect bogie structure`s reliability and passenger`s comfort. In view of this, some scholars from

\author{
Guan-Hua Huang \\ State Key Lab of Traction Power, Southwest Jiaotong \\ University, Chengdu 610031, Sichuan China) \\ E-mail: maklcewxy@163.com
}

china and foreign countries had launched lots of research about the impact of polygonal wheel, the main research focused on the impact of wheel polygon with vehicle dynamic performance[2-7]. Overall, the current research on polygonal wheel mainly concentrated in the formatting principle of polygonization and impaction of dynamic performance, however, research in other fields were seldom mentioned.

Just because the gearbox is the key component of high-speed train, according to lots of failure gearbox during operation, technical analysis also make sure that the wheel polygon has an inevitable impact on connection with these failure $^{[8]}$. Therefore, no matter what points of view from academic research or engineering practice, it will be great realistic significance to study the influence between polygonal wheel excitation and dynamic characteristics of high-speed train gearbox.

\section{MODEL OF POLYGONAL WHEEL}

There are a lot of mathematic methods can be used to simulate polygon of wheel, a traditional method is to expand wheel radial deviation into Fourier transform function periodically, and which be equivalent superimposed into the track's geometric irregularity, wheel profile don't need been

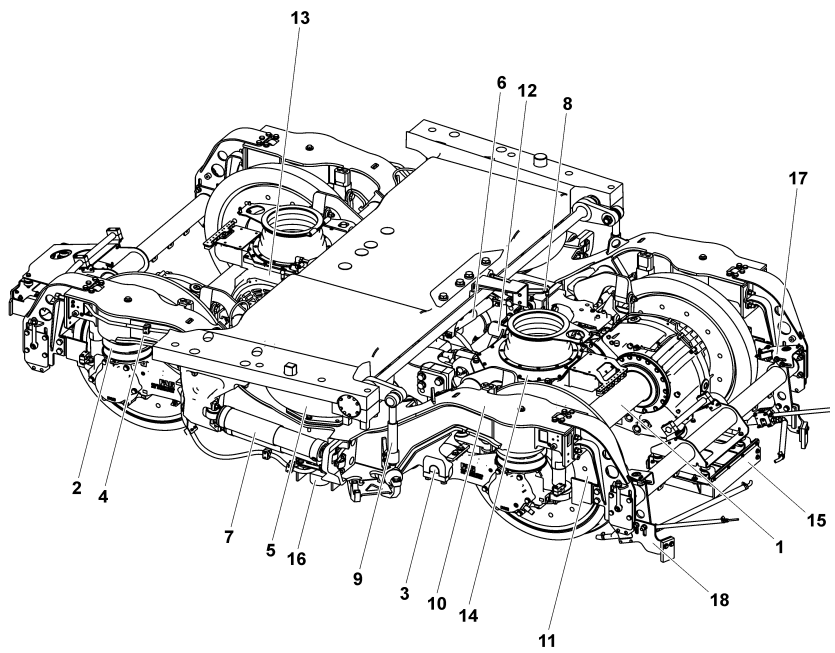

1. Wheel-sets; 2. Primary suspension;3. Axle-box positioning device;4. Lateral stopping block; 5. Secondary suspension; 6. Lateral damper;7. Anti-snake movement damper; 8. Air spring connecting rod; 9. Anti-rolling resistant torsion bars; 10. Motor bogie; 11. Brake unit; 12. Drawbar unit;13. Driving system; 14 . Motor wind aisle; 15 . ATP;16 Induction receiver; 17 .Wheel flange lubrication device.

Figure 1. Structure diagram of a kind of EMU motor bogie. 
changed. Another method is aim at the circumference of the wheel, using the method of harmonic to simulate, the calculation equation as follows[8]:

$$
\left\{\begin{array}{l}
\Delta r=A \sin \left(n \beta+\beta_{0}\right) \\
r(\beta)=R-\Delta r \\
\beta(t)=\beta(t-1)+\omega \Delta t
\end{array}\right.
$$

In the above formula (1):

$A$ - wave depth, it equal to the amplitude of polygonal wheel;

$n$ _orders of polygonal wheel, it refers to the harmonic cycles that format a radius difference between actual wheel radius and nominal wheel radius within $360^{\circ}$ direction;

$\beta$-Wheel rotation angle; $\beta_{0}$-Phase angle;

$\Delta r$-Wheel diameter difference along the circumference direction;

$R$-Nominal radius of wheel rolling circle

By changing polygon orders and wave depth of different wheel, we can simulate different working conditions with different polygonal wheels.Figure2 represents cyclical irregularity of wheel in the circumference direction, which was defined by using harmonic method, in this figure2: (1) the red curve represent the irregularity of the third order, (b) the red curve represent the irregularity of the twentieth order

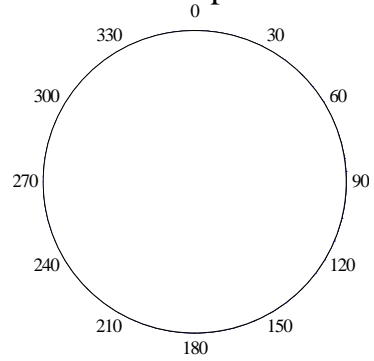

(a) The third order irregularity

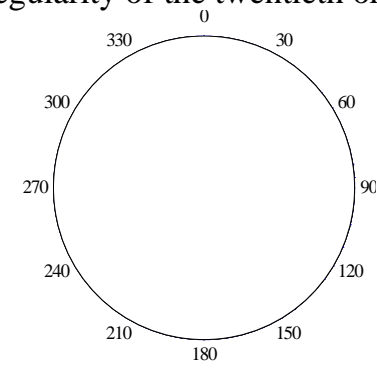

(b) The twentieth order irregularity
Figure 2. The corresponding curves of orders and shape of polygonal wheel.

\section{MESHING MODEL OF GEAR PAIR}

Comparing with the general mechanical transmission system, gearbox system of high-speed train is relatively simple in the structure aspect, which has only single reduction gear pair, despite it owns simple structure, however, considering the complexity of wheel/rail contact and high running speed, the vibration response of gear system is still very complex during high-speed train operating process. The article based on the multi-body dynamics software SIMPACK to set up whole vehicle model of High-speed train, and carried on a serious of systemic analysis. For the modeling methods of gear pair, there are two basic force elements: the traditional modeling method is the 14th force element, which only consider the equivalent torque among the driving shaft and following shaft, this method had been used in some past articles, this equivalent method doesn't need to consider dynamic meshing characteristics and transmission elasticity within gear pair, which is simple modeling method; The second method is the $225^{\text {th }}$ force element, which utilize flexible penetration depth on tooth and contact stiffness to calculate meshing force between tooth pair, meanwhile, this method also considered the coulomb friction between teeth as well as normal damping during contacting. Thus, to use force elements need more detail gear parameters and material parameters, at the same time, this force element can research center distance of gear, status changing of rotation shaft as well as tooth profile modification. Because this article needs to consider vibration characteristics of driving system under eternal incentives and internal incentives, therefore, the $225^{\text {th }}$ force element has been used to establish model. Table1 represents basic parameters of gear pair transmission, Figure3 refers to dynamic model of gear meshing.

TABLE I. TABLE THE GEOMETRY PARAMETERS OF GEAR PAIR

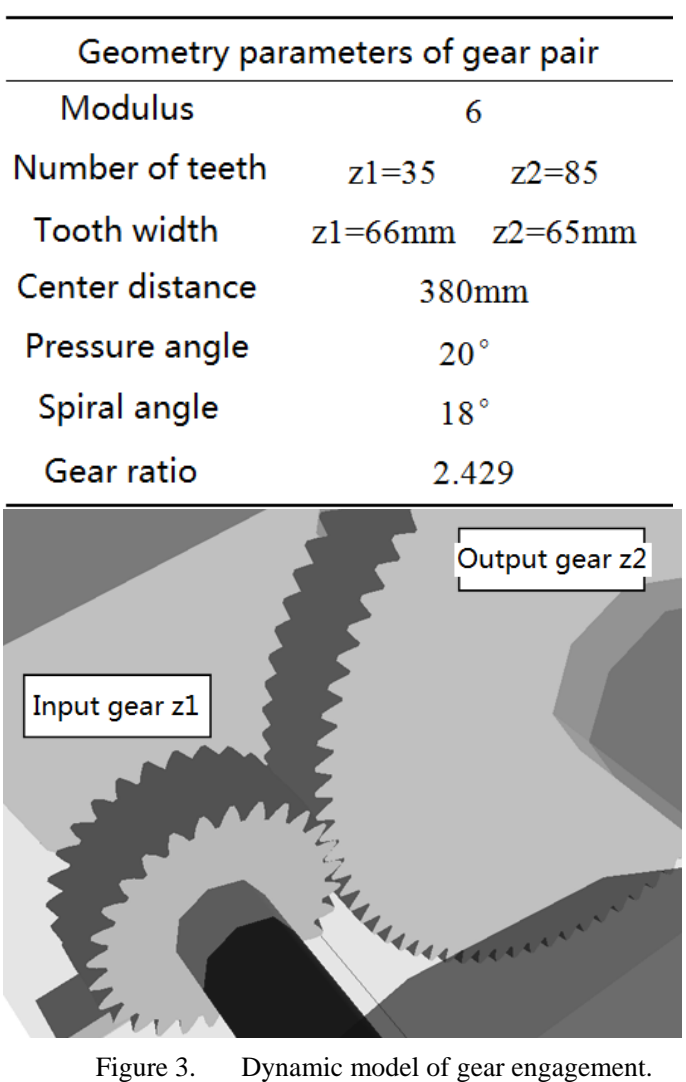

\section{DYNAMICS MODEL OF VEHICLE SYSTEM}

In order to considered the influence of vehicle system coupling action on gearbox, so the SIMPACK software of multi-body dynamics is used to establish the dynamic model of whole vehicle, which included 86 DOFs (degree of freedom) (see Table2)[9], the dynamic calculation model including 23 rigid bodies, for example: car body, bogie frame, wheel-set, motor, gearbox , axle box and etc., gearbox included housing, output gear and input gear. Motor was installed on the bogie frame, which has respective 3 rotational DOF and 3 axial DOF, car body and 
bogie frame have 6 DOF respectively, each one axle box has only 1 DOF. The wheel set has 4 independent DOF, these DOFs involved up, down and roll of wheel set are and hydraulic damper, between car body and bogie frame are equipped with horizontal damper and stop, meanwhile, the nonlinear factors has been considered. restrained by nonlinear contact geometry relationship of wheel/rail. Wheel set and bogie frame using rotation arm node to localize, and equipped with primary vertical springs Wheel tread profile used S100CN, rail profile used CN60 rail, Figure4 is the dynamics model of whole vehicle.

TABLE II. TABLE DOF OF VEHICLE SYSTEM

\begin{tabular}{|c|c|c|c|c|c|c|c|}
\hline \multirow[b]{2}{*}{ Components } & \multicolumn{6}{|c|}{ DOF } & \multirow[b]{2}{*}{ remalks } \\
\hline & longitudinal & hoizontal & Vetical & Rolling & Nodding & Shodking & \\
\hline Body & $X_{c}$ & $Y_{c}$ & $Z_{c}$ & $\Phi_{c}$ & $\theta_{c}$ & $\psi_{c}$ & \\
\hline Frame & $X_{s i}$ & $Y_{s i}$ & $Z_{s i}$ & $\Phi_{s i}$ & $\theta_{s i}$ & $\psi_{s i}$ & $i=1-2$ \\
\hline Wheetset & $X_{w j}$ & $Y_{w j}$ & $Z_{w j}^{*}$ & $\Phi_{w j}^{*}$ & $\theta_{w j}$ & $\psi_{w j}$ & $j=1-4$ \\
\hline Motor & $X_{m j}$ & $Y_{m j}$ & $Z_{m j}$ & $\Phi_{m i}$ & $\theta_{m j}$ & $\psi_{m j}$ & $j=1-4$ \\
\hline Axlebox & & & & & $\theta_{a i}$ & & $i=1-8$ \\
\hline Gearbox & & & & & $\theta_{b i}$ & & $i=1-4$ \\
\hline Ouputgear & & & & & $\theta_{b b i}^{*}$ & & $i=1-4$ \\
\hline Inputgear & & & & & $\theta_{b s i}$ & & $i=1-4$ \\
\hline
\end{tabular}

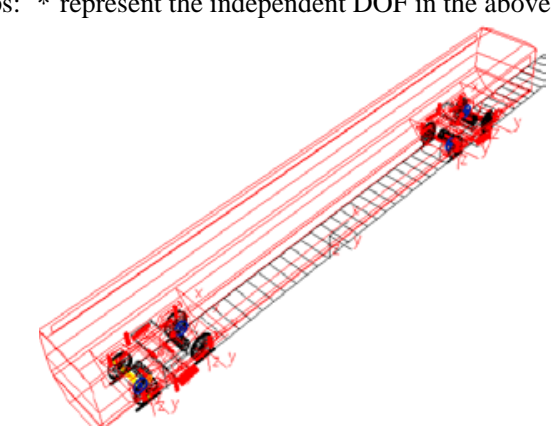

Figure 4. Dynamics model of vehicle system.

\section{ANALYSIS RESULTS OF KINETICS}

According to the investigation results, during the process of High-speed train operating, the polygonal wheel is not only performance for the first order polygon, it`s very often will combine a variety of conditions of polygon [10]. Thus, this paper adopted an approach of multiple harmonic inputting to spread a variety of polygonal conditions, these polygonal working conditions contain Fourier series of different harmonic forms, so as to simulate the influence of actual radial deviation on gear transmission system. According to the testing results, this paper mainly consider these influence of polygonal wheel which consists of: the first order, the third order, the sixth order, the $11^{\text {st }}$ order, the $15^{\text {th }}$ order, the $18^{\text {th }}$ order and the $20^{\text {th }}$ order, assumed their wave depth are respective $0.01 \mathrm{~mm}, 0.02 \mathrm{~mm}, 0.1 \mathrm{~mm}, 0.05$ $\mathrm{mm}, 0.06 \mathrm{~mm}, 0.05 \mathrm{~mm}$ and $0.05 \mathrm{~mm}$. The train`s speed of simulation is $300 \mathrm{~km} / \mathrm{h}$, the figure 5 shows the gear transmission system`s response that has been calculated. We can find that, under the combination conditions of more orders polygons, the vibration of gear transmission was increased significantly, in order to analyze contribution of each order frequency on vibration, we took the actual online test results as example to analyze frequency spectrum(see Figure 6), we can see that the gear transmission system including the meshing frequency component $(2346 \mathrm{~Hz})$ from dynamic incentive was owned by itself, also including other polygon incentive $431 \mathrm{~Hz}$ (the $15^{\text {th }}$ order), $516 \mathrm{~Hz}$ (the $18^{\text {th }}$ order), $574 \mathrm{~Hz}$ (the $20^{\text {th }}$ order), the calculation formula of frequency as follows(2), compare to low orders polygon incentive, the more orders, the greater influence on gear transmission system.

$$
f=N v /\left(2 \pi R_{i}\right)
$$

In above formula, $N$-the order of polygonal wheel, $v$-train speed, $R_{i}$-rolling circular radius of polygonal wheel along circumferential direction. 


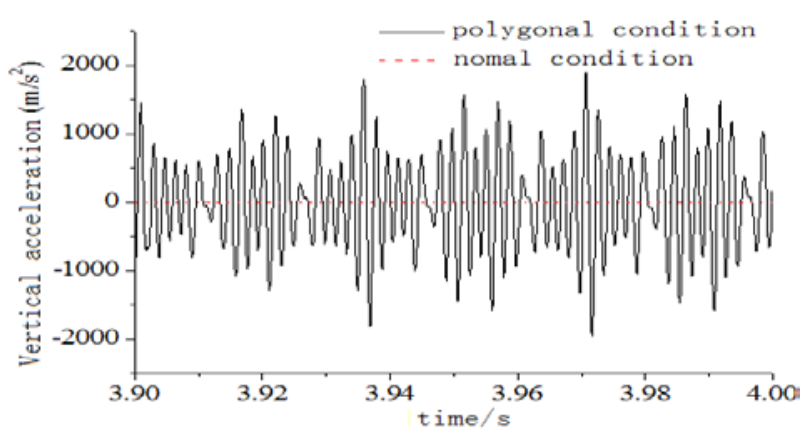

(a) Vertical acceleration of gearbox

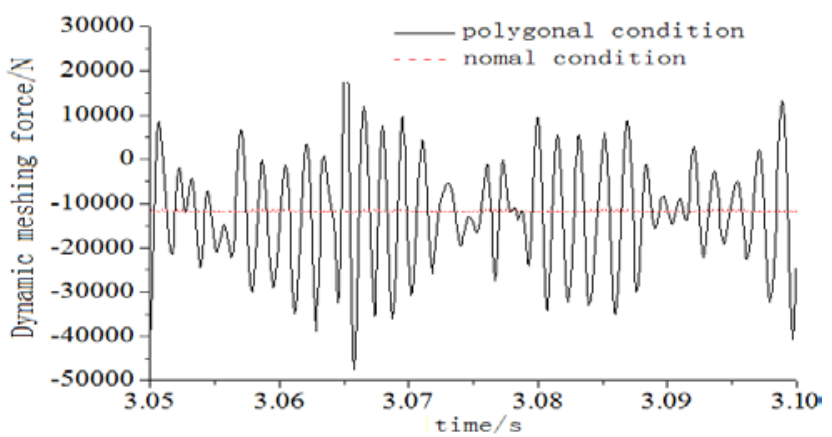

(b) Dynamic meshing force of gear pair

Figure 5. The influence on gear vibration of polygonal wheel.

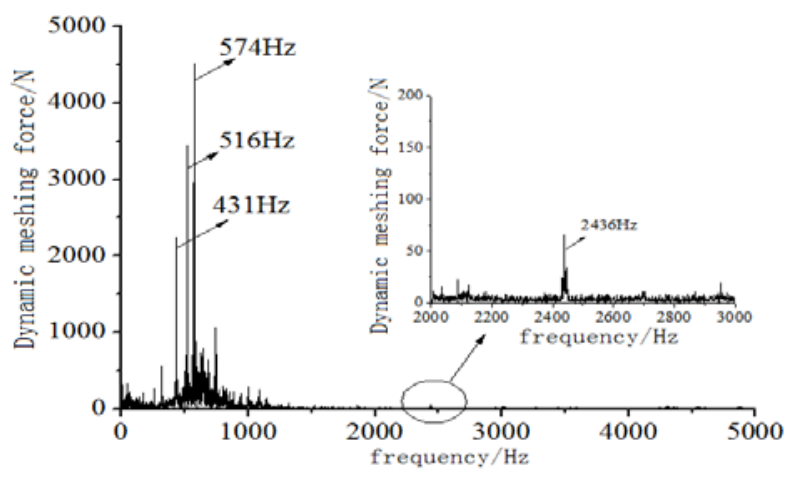

Figure 6. The frequency spectrum of gear meshing force.

\section{ANALYSIS OF DYNAMIC CHARACTERS}

This paper takes High-speed train`s gearbox as an example, carried on analysis of housing finite element, also used solid elements to mesh model(see figure7), we got 363525 units and 97786 nodes. The housing material is casting aluminum alloy, the allowable stress is $210 \mathrm{Mpa}$, material density $\rho=2.7 \times 10^{3} \mathrm{~kg} / \mathrm{m} 3, \quad E=75$ Gpa, Poisson's ratio $=0.3$. In order to calculate the constraint modal of gearbox, set the constrained boundary condition is: set fixed constraint on the connection surface between the input end and $\mathrm{C}$ type bracket, bearing hole of output end used constrained DOF except for around the axle rotational degree of freedom. Got housing model frequency before 14 orders as shown in table3.

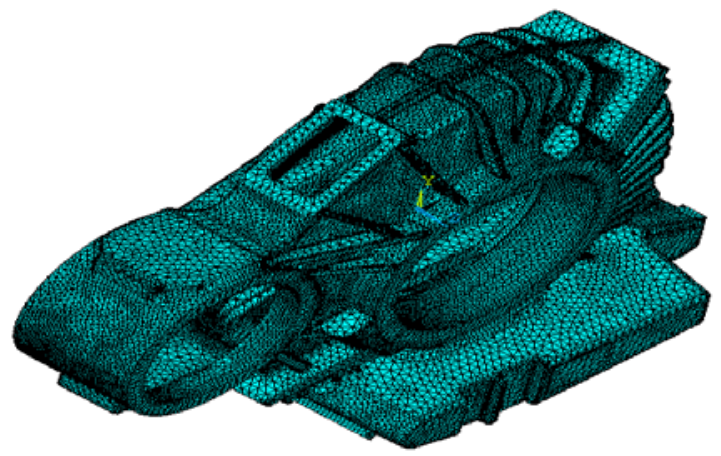

Figure 7. Finite element mode of housing.
TABLE III. TABLE HOUSING MODEL FREQUENCY BEFORE 14 ORDERS

\begin{tabular}{cccc}
\hline orders & frequency & orders & frequency \\
\hline 1 & 5.24 & 8 & 908.09 \\
2 & 14.21 & 9 & 1031.9 \\
3 & 210.42 & 10 & 1084.2 \\
4 & 579.12 & 11 & 1149.2 \\
5 & 754.88 & 12 & 1194.4 \\
6 & 829.79 & 13 & 1249.4 \\
7 & 852.86 & 14 & 1337.9 \\
\hline
\end{tabular}

According to dynamics analysis results in section 4, apply the dynamic force to gearbox housing finite element model, based on the above constraints, carried on transient analysis, we can get displacement, velocity and acceleration of each node of housing. This paper utilize ANSYS to calculate finite element model, considering the meshing frequency is very high, so the sampling frequency is also very high, consequently, the calculation time of finite element used 0.1second, step time used 500steps. Figure 8 represents the maximum stress trend of housing, Figure 9 represents frequency response curve of corresponding points of housing. From the below two figures we can see that the two spectrum diagram have main vibration frequency $574 \mathrm{~Hz}$, which close to the housing`s inherent frequency $579.12 \mathrm{~Hz}$ (the $4^{\text {th }}$ order), meanwhile, the $574 \mathrm{~Hz}$ is corresponding to the rolling frequency of the $20^{\text {th }}$ order polygonal wheel, in other words, this result can explain that when the excitation frequency of polygonal wheel is closely to natural frequency of housing, this situation will lead to increase stress of housing as well as reduce life of housing.

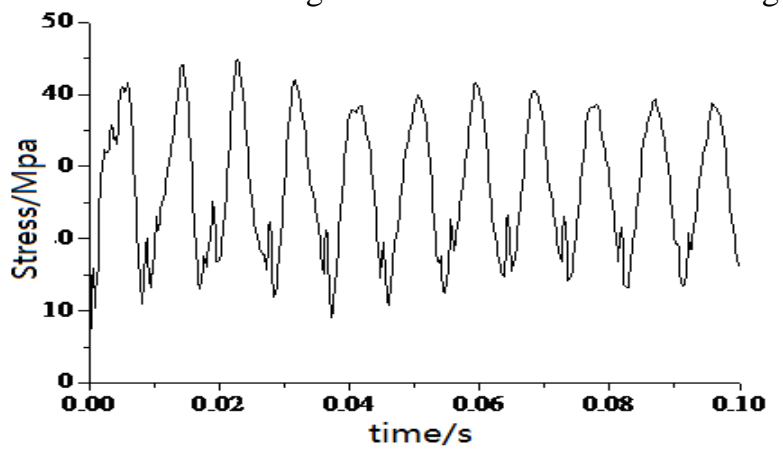

Figure 8. The maximum dynamic stress variation. 


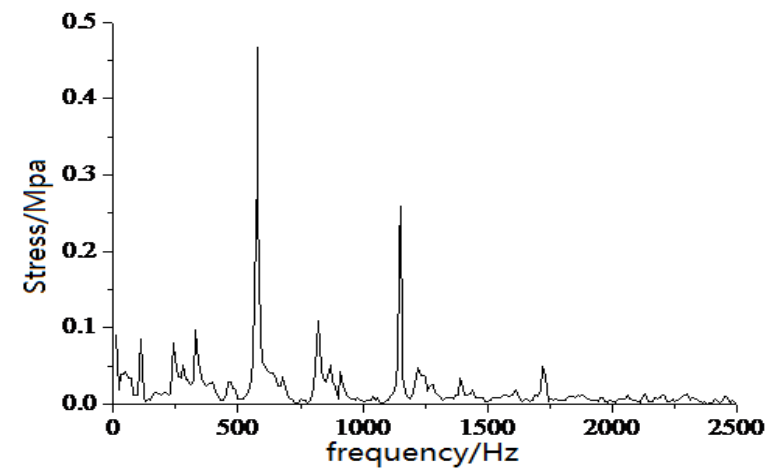

Figure 9. Dynamic stress frequency of housing nodes.

\section{CONCLUSIONS}

Through simulation calculation and systemic analysis, this paper can get the following conclusions:

(1)During the process of high-speed train operating, the gearbox system will bear not only the external turbulence form wheel/rail, but also from gear meshing incentives.

(2)Polygonal wheel will influence to transmission system`s performance significantly, the incentive of polygonal wheel will lead to obvious increase of gearbox system`s vibration.

(3) Polygonal wheel will influence to dynamic performance of gearbox housing, when the polygon excitation frequency close to the inherent frequency of housing, it`s easily to cause resonance, which will affect the limit of fatigue life of housing eventually.

\section{REFERENCES}

[1] Weihua Zhang. Whole EMU and bogie [M]. BeiJing: China railway publishing house,2011.

[2] Wu Lei, Zhong Shuoqiao, Jin Xuesong, etc,. Influence of polygonal wheel on running safety of vehicle[J]. Journal of traffic and transporation engineering. 2011,11(3) :47-54

[3] Cui Dabin, Liang Shulin, Song Chunyuan, etc,. Out of round high-speed wheel and its influence on wheel/rail behavior[J].Journal of mechanical engineering,2013,49(18):8-16.

[4] Zhang Xueshan, Xiao Xinbiao, Jin Xuesong. Effect of ovalization of high speed railway wheels on vehicle dynamic performance[J]. Journal of mechanical engineering,2010,46(16):67-73.

[5] Zhang Xueshan, Xiao Xinbiao, jin Xuesong. Influence of high speed railway wheels ovalization on vehicle lateral stability[J]. Journal of mechanical engineering, 2008,44(3):50-56.

[6] Wang Yijia, Zeng Jing, Luo Ren, etal. Effect of polygonal wheel on vehicle dynamic performance[J]. Journal of Sichuan University (Engineering Science edition), 2013,45(3):176-182.

[7] Wang Xingyu, Fan Jun. Research on Relation between Interior Noises and Out-of-round Wheel of High-speed EMU[J].Journal of The China Railway Society, 2013,35(9):14-18.

[8] Yongguo Deng.Effect of Out-of-round Wheel on Vehicle System Dynamics Behavior [D].ChengDu, Southwest JiaoTong university, 2014.

[9] Huang Guanhua,Wang Xingyu. Dynamic Response Analysis of Gearbox Housing System Subjected to Internal and External Excitation in High-speed Train[J]. Journal of mechanical engineering, 2015,51(12):95-100.

[10] Luo Ren, Zeng Jing, Wu Pingbo . Simulation and analysis of wheel out-of-roundness wear of high-speed train [J]. Journal of the china railway society, 2010, 32(5):30-35. 